\title{
EVALUATION OF DISCOMFORT GLARE IN THE 50+ ELDERLY: EXPERIMENTAL STUDY
}

\author{
AGNIESZKA WOLSKA ${ }^{1}$ and DARIUSZ SAWICKI ${ }^{2}$ \\ ${ }^{1}$ Central Institute for Labour Protection - National Research Institute (CIOP-PIB), Warszawa, Poland \\ Department of Safety Engineering, Laboratory of Optical Radiation \\ ${ }^{2}$ Warsaw University of Technology, Warszawa, Poland \\ Institute of Theory of Electrical Engineering, Measurements and Information Systems
}

\begin{abstract}
Objectives: The aim of the study was to analyze discomfort glare sensation in subjects aged 50 years and more in comparison with younger subjects (i.e. younger than 35 years of age). Material and Methods: The experiments were performed on a computer workstation placed in controlled lighting environment where 2 discomfort glare conditions were modeled. Each participant performed for about $1 \mathrm{~h}$ specially designed visual tasks, including the tests with Landolt's rings presented on the screen by a computer program. The glare evaluation method consisted of subjective evaluation of discomfort glare on the semantic glare rating scale, tests of mesopic contrast and glare sensitivity, subjective assessment of lighting quality and asthenopic symptoms. The time needed to perform the task and the number of mistakes were also recorded. The subjective evaluation of glare was compared with the Unified Glare Rating (UGR) index calculated by the DIALux simulation program. Results: A higher percentage of the younger group subjects assessed glare after the experimental session as uncomfortable and intolerable than in the 50+ group, who more often assessed glare as acceptable. The assessment of discomfort glare in the younger group corresponded to higher UGR value compared to UGR value calculated by DIALux. In the $50+$ group, such correlation was found only for lower discomfort glare (UGR = 19). The results showed that younger participants more frequently suffered from visual fatigue and assessed lighting as less comfortable. However, the mesopic glare sensitivity increased significantly after the experiments only in the 50+ group under both glare conditions. Conclusions: The obtained results showed that discomfort glare sensation changes with age. The younger population seems to be more sensitive and demanding than the older one in relation to discomfort glare limiting, in spite of the lack of significant objective measures of fatigue. The exposure of the elderly to bigger discomfort glare could adversely affect the objective measures of fatigue like mesopic glare sensitivity and visual performance.
\end{abstract}

Key words:

Lighting, Glare, UGR, 50+ elderly, Asthenopia, Discomfort glare evaluation

\section{INTRODUCTION}

According to the WHO definition, "Health is a state of complete physical, mental and social well-being and not merely the absence of disease or infirmity" [1]. This definition highlights the important aspects of well-being and disorders, which could be caused also by different environmental agents. A very important physical agent which exists in all workplaces is lighting.
Vision depends on light and, consequently, lighting installations can contribute both positive and negative effects in humans. Lighting influences the individual well-being. In the lighting quality model, individual well-being is understood as human needs related to lighting that is appropriate to maintain good health, as well as lighting for visibility, task performance, visual comfort, esthetic judgment, and also interpersonal communication [2,3]. This model confirms

Received: October 10, 2013. Accepted: February 5, 2014.

Corresponding author: D. Sawicki, Warsaw University of Technology, Institute of Theory of Electrical Engineering, Measurements and Information Systems, Koszykowa 75, 00-662 Warszawa, Poland (e-mail: dasa@iem.pw.edu.pl). 
systemic effects of light on humans. Light through the eye can influence human physiology, mood and behavior $[3,4]$. This is why lighting should provide an environment in which people, through the sense of vision can work effectively, efficiently and comfortably [5]. The role of lighting designers is to optimize the visual environment taking into account: visual performance, comfort and appearance.

Glare is one of the parameters which decide about the quality of lighting. It is "the sensation produced by luminance within the visual field that is sufficiently greater than the luminance to which the eyes are adapted to cause annoyance, discomfort or loss in visual performance and visibility" [5]. There are 2 main forms of glare: disability glare and discomfort glare. Disability glare is a visual performance effect, and discomfort glare is a comfort and health effect. Each one is associated with particular glare sources [6]. Disability glare impairs the vision of objects as a result of stray light in the eye, which produces veiling luminance on the retinal image and that way reduces its contrast. As a consequence, the visual performance and visibility are reduced. Discomfort glare produces discomfort due to an unsuitable distribution or range of luminance or to extreme contrasts in space or time [7]. However, this form of glare does not necessarily interfere with visual performance or visibility [5].

The results of many studies have suggested a physiological basis for discomfort glare complaints [8], and although the precise mechanism is unknown [6], discomfort glare refers to a subjective impression of discomfort. Nevertheless, both forms of glare influence human well-being and also could be the risk factor in the working environment [9]. The limitation of this phenomenon is the goal of researchers and lighting designers.

Disability glare is relatively easier to investigate by assessing the visual performance with and without the presence of the glare source, but the quantification of discomfort glare is much more difficult. In ordinary interior lighting installations, the control of discomfort glare is much more important than the control of disability glare, because if discomfort glare is adequately limited, disability glare will not exist [7]. However, as known from the lighting practice, the adequate limitation of discomfort glare is often hard to obtain.

Numerous studies dedicated to discomfort glare have been conducted, but the psycho-physiological origin of that phenomenon is still not very well understood. The methods of objective assessment of discomfort glare have been investigated. On the one hand, the activity of frontalis muscle and the diameter of the pupil did not correlate with subjective evaluation of discomfort [10], but on the other hand, the results of EMG measurement of electrical activity associated with facial muscles correlated with the subjective assessment of discomfort glare [8]. There may be multiple factors contributing to these results, including the procedural ones. Both the explanation of glare sensation to the subjects and the used scale of glare sensation may affect the results of subjective evaluation [11-14].

In spite of the above, the subjective evaluation of discomfort glare is still the main measure and the results of many studies have given the grounds to establish the correlation between sensation of glare and factors controlling discomfort glare. Discomfort glare depends mainly on glare source luminance, but also on background luminance (which controls the adaptation level of the observer's eye), solid angle subtended by the source at the observer's eye and angular displacement of the source from the observer's line of sight [7].

There are different predictive models of glare evaluation, which calculate the glare constant value (numerical value) based on lighting installation parameters. The numerical values of glare correspond with subjective sensation of glare on a semantic scale. The most often used scale of glare sensation is the multi-criterion Hopkinson system $[7,15]$. This way, the semantic glare scale is transformed into the numerical scale [7]. Currently, the Unified Glare Rating (UGR) formula predictive model is dominant and recommended by the CIE standard for lighting 
of interior workplaces [16] and it was implemented in the European Standard EN 12464-1 [17]:

$$
\mathrm{UGR}=8 \log \left(\frac{0.25}{\mathrm{~L}_{\mathrm{u}}} \sum_{\mathrm{i}} \frac{\mathrm{L}_{\mathrm{i}}^{2} \omega_{\mathrm{i}}}{\mathrm{P}_{\mathrm{i}}^{2}}\right)
$$

where:

$\mathrm{L}_{\mathrm{u}}$ - background luminance ( $\left.\mathrm{cd} / \mathrm{m}^{2}\right)$,

$\mathrm{L}_{\mathrm{i}}$ - luminance of glare source $\mathrm{i}$ in the direction of the observer's eye $\left(\mathrm{cd} / \mathrm{m}^{2}\right)$,

$\omega_{i}$ - solid angle of the glare source i seen from the observer's eye (sr),

$\mathrm{P}_{\mathrm{i}}$ - position index (Guth's index) for the glare source i according to Guth's analysis.

The UGR factor is a psychological parameter intended to assess any adverse subjective discomfort response to lighting environment containing glare sources.

In practice, it is difficult to calculate the UGR value according to formula 1 . There are 2 known effective methods: simulation of the luminance distribution using proper computer software or measurement of the luminance with an array photometer [18]. In the presented study, the simulation program was used.

The glare rating scale is an interval. The differences between the numbers represent the perceptible differences in sensation of glare. One glare rating unit is the least detectable step and 3 units make an acceptable step in glare criteria [19]. The theoretical range of the UGR scale is from 10 to 30 [19], but the practical range used in the European standard EN 12464-1 [17] is from 16 to 28 $(16,19,22,25,28)$. High values of UGR correspond to significant discomfort glare, while a low value indicates little discomfort glare. There are no official relationships between the UGR value and the point on the semantic discomfort glare scale. For example, UGR $=19$, which is the maximum permissible value for office work, could be related to just acceptable glare sensation [14], to unacceptable glare [13] and, in the opinion of the Authors of this article, also, to acceptable glare.
The European lighting standard EN 12464-1 [17] presents the requirements and recommendations for lighting parameters for different workstations and activities. The requirements for glare limitations are based on UGR values, which should not be exceeded for particular work task/activity. There are no requirements distinctions between UGR values for young and older workers, however age-related changes in vision are well known. Especially the lens thickens and is becoming more opaque with age. This is the reason of a decrease in spectral transmission through the human lens and a reduction in accommodative power. Light entering the eye of the elderly is more scattered as a result of optical imperfections in the eye and less light reaches the retina $[3,5]$. These age-related changes have not been taken into account in the recommendations of lighting standards [17] yet. Despite the general statement of increasing the level of illuminance for people of vision below normal, there are no recommendations for the lighting parameters adequate for the older population.

It is well known that the world population is rapidly ageing. This also brings new changes resulting in extended working years. However, the physical capacities and needs of the elderly are not the same as those of the younger population, so many studies have been focused on how to make up for such differences. The key aspect is adaptation on all levels: individual, organizational and societal and it is associated, among other things, with the adjustment of the working environment (including lighting) to the psycho-physiological capacities of the elderly.

The studies dedicated to the lighting for older people concerned mainly many aspects of local lighting [20-22], and aspects of increasing the level of illuminance $[20,23,24]$ or the color of light [25]. Taking into account that some studies pointed out that elderly people are more sensitive to disability glare [24-26], it would be worth taking into consideration discomfort glare and determining the possible changes in lighting recommendations for the UGR value. 
The aim of the article is to present the results of a study on discomfort glare sensation in subjects aged 50 years and more in comparison with younger subjects (i.e. 35 years old and younger) carried out in a laboratory room under controlled lighting environment for 2 modeled discomfort glare conditions.

\section{MATERIAL AND METHODS}

\section{Study design}

The study was performed in a lighting modeling laboratory situated in the Central Institute for Labour Protection National Research Institute in Poland. It was conducted under 2 modeled discomfort glare conditions from lighting installation. Working at the computer, as an example of typical office work where discomfort glare should be especially controlled, was chosen for the experiments. On the basis of the UGR calculations by a lighting simulation program - DIALux [27], 2 different observer's positions in the room in relation to the glare sources were chosen. First, at
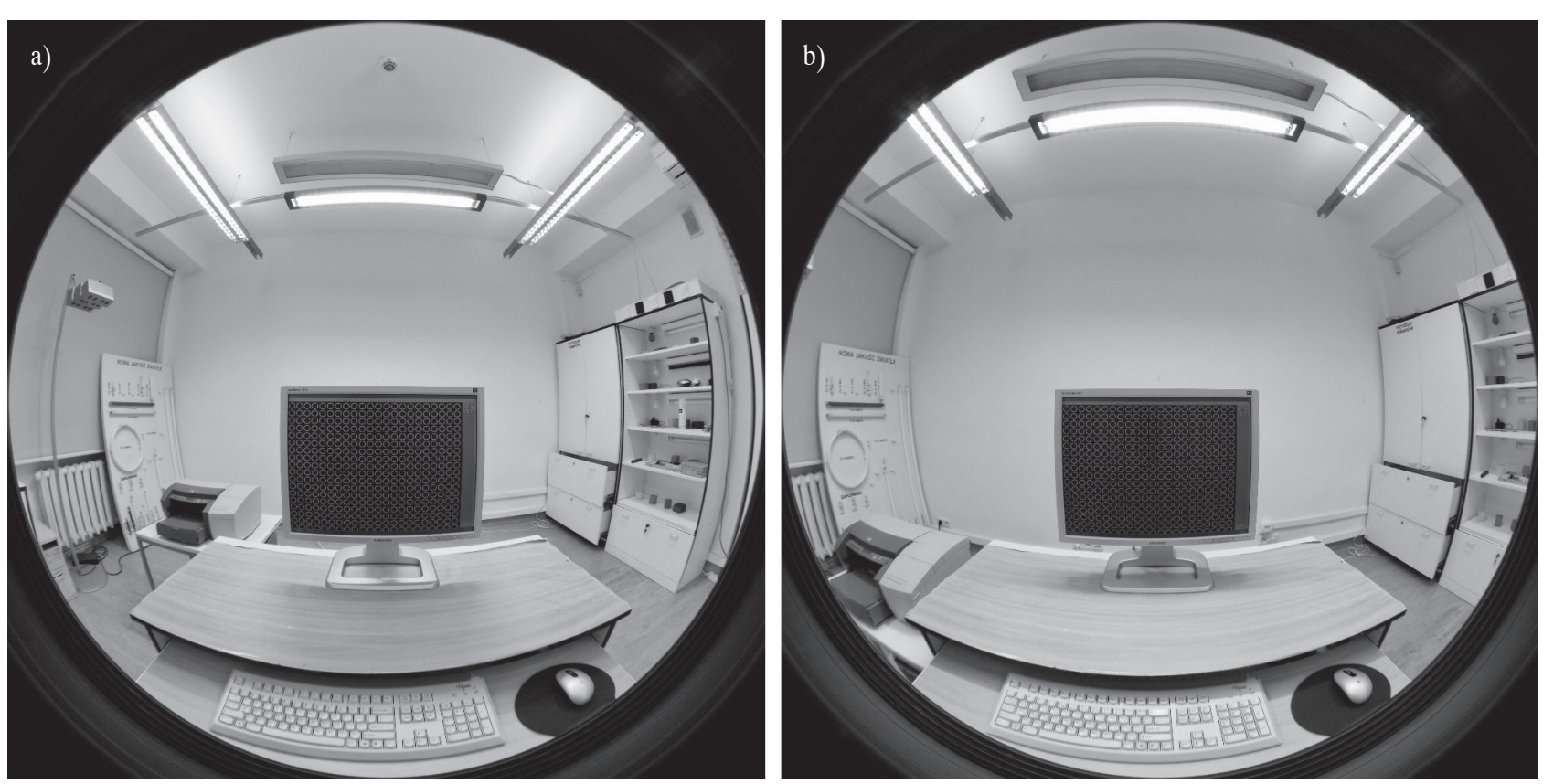

which UGR = 19 (i.e., the limit value of UGR for office work), and second for higher discomfort glare conditions at which UGR = 22 (i.e., higher by 3 units, which corresponded to the acceptable step in glare criteria [19]). In the visual field of the observer, there were 3 luminaires, which constituted the glare sources (Photo 1). One luminaire: matt mirror ribbed lamellae louver TCS $2602 \times 54 \mathrm{~W} \mathrm{M2,}$ manufactured by Philips Lighting. Two luminaires: dark light louver TECTON-D-ID $2 \times 35 \mathrm{~W}$, manufactured by Zumtobel.

Fifty participants took a part in the experiments. Before the experiments the participants were trained in performing the visual tasks. They were also provided with explanations concerning the semantic glare rating scale and trained in glare assessment according to that scale. Each participant took part in 2 experiments for both glare conditions, but the experimental sessions were carried out on 2 different days. The subjects in both groups were randomly divided into 2 subgroups. Each subgroup started performing the 1st experiment in different discomfort glare conditions.

Unified glare rating: a) 19, b) 22.

Photo 1. View of glare sources in the observer's visual field for the modeled discomfort glare conditions 
The aim was to eliminate the memorization effect on the results. The procedure for the experiment was developed on the basis of a pilot study. The glare evaluation method consisted of subjective evaluation of discomfort glare on the semantic glare rating scale, tests of mesopic contrast and glare sensitivity, subjective assessment of lighting quality and visual fatigue. Additionally, visual performance was evaluated by recording the time needed to perform the task and number of mistakes.

The visual task was designed for the typical office work activities taking into consideration the main locations of the visual line of sight directed on the screen, document and keyboard. Computer screen was the main object of observation and the visual task made the observers fix their eyesight on the screen throughout most of the time of the experimental session, because in that position the glare sources were in the visual field of the observers. During keyboard or document observation, glare sources were out of the visual field, but these tasks were implemented mainly for the sake of differentiation of the subject's activity and changing the position.

\section{Subjects}

The subjects were selected according to the criteria of age (50+ group - subjects above 50 years old, control group subjects 19-35 years old) and the eyes state. The volunteers had to undergo the ophthalmological examinations before classification to experimental groups. The aim of these examinations was to exclude subjects with potential visual disabilities or illnesses, which could influence the results of glare evaluation. The subjects selection criteria were as follows: myopia ( $\leq 8 \mathrm{Dsph}$ ), hyperopia ( $\leq 5 \mathrm{Dsph}$ ), astigmatism $(\leq 2.5 \mathrm{Dcyl})$, refractive errors corrected by glasses or contact lenses, appropriate color vision, no abnormalities in the examination of the anterior segment or the fundus of the eyes, no coexisting diseases of the sight organ (e.g. systemic diseases like diabetes, arterial hypertension) and no eye surgeries. As a result of ophthalmologic examinations, 50 participants were qualified to this study. The characteristics of both groups are presented in Table 1.

\section{Visual task}

The visual task consisted of 3 types of office work, i.e.: comparison of the printed text with the text presented on the screen, rewriting the printed text into computer word editor, and performing the tests with Landolt's rings presented on the screen by a specially prepared computer program.

The visual task on the screen was simulated by a computer program. Such method was successfully used in previous studies $[28,29]$. That task consisted of 5 Landolt's tests ( 3 with negative polarity and 2 with positive polarity).

Table 1. Group characteristics

\begin{tabular}{|c|c|c|}
\hline Feature & $50+$ group & Control group \\
\hline Participants (n) & 29 & 21 \\
\hline women & 26 & 13 \\
\hline men & 3 & 8 \\
\hline \multicolumn{3}{|l|}{ Age (years) } \\
\hline $\mathrm{M} \pm \mathrm{SD}$ & $57.9 \pm 4.64$ & $24.9 \pm 3.95$ \\
\hline $\min .-\max$ & 51-69 & 19-34 \\
\hline Participants wearing correction glasses/lenses (n) & 27 & 10 \\
\hline
\end{tabular}

$\mathrm{M}$ - mean; SD - standard deviation.

min. - minimal value; max - maximal value. 
Each test consisted of 396 white or black rings in eight positions of the gap.

There were 3 kinds of Landolt's tests: a "step by step" test (the gap of each displayed ring had to be identified and marked), an "option" test (only 3 selected gap positions had to be fond, identified and marked), and a "perceptivity" test (a ring, randomly highlighted for $2 \mathrm{~s}$, had to be noticed, identified and marked). The gap localization was indicated by clicking an arrow related to its position. Each of these tests had a different distribution of the rings' gaps on the screen. The computer program registered the number of mistakes and the time needed to perform the visual task, which could be the measure of visual performance.

\section{Subjective evaluation}

The subjective evaluation concerned: discomfort glare, lighting quality and visual fatigue. The evaluation of discomfort glare was carried out before the visual tests and just after finishing the tasks using the visual analog scale. The semantic discomfort glare scale was elaborated for the purpose of this study on the basis of the Hopkinson scale [10], with 3 demarcations positioned to signify the borders between just acceptable, just uncomfortable, and just intolerable discomfort (presented in Figure 1). A few days before the experimental session, the subjects were explained and trained in the use of the glare sensation rating scale. The position on the Hopkinson's scale [10] that is related to just perceptible discomfort was omitted, because of the pilot study results (subjective assessment of glare started from just acceptable) and DIALux calculations of UGR in the examined observer's locations (no stated UGR values, which are related to the sensation of lower glare than just acceptable).

The subjective evaluation of the lighting quality was performed by a specially prepared questionnaire which consisted of 5 questions. The answers were marked on the ordinal scale from 1 to 5 , where 1 referred to the assessment

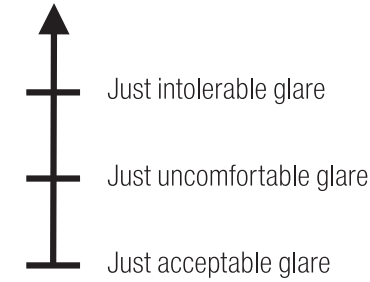

Fig. 1. Discomfort glare rating scale used in the experiments of lighting as poor and 5 - as very good. The questions concerned the following aspects: the illumination level on the working plane, visual comfort related to lighting, experienced annoyance related to bright elements of glare sources, declaration of how frequent the participant could work in the modeled lighting conditions and acceptance of that lighting for permanent work. The questionnaire was filled in after the experimental session.

Visual fatigue manifests itself in the weakening of a variety of visual functions and in the form of specific complaints reported by persons who perform a strenuous visual task. The subjective evaluation of visual fatigue was established by an asthenopic symptoms questionnaire, which consisted of questions on the following symptoms: itching, tearing, oversensitivity to light, blurring, visual tiredness, headache, heaviness of eyelids, redness, shooting pain. The answers were marked on the ordinal scale from 1 to 5 , where 1 meant "no complaint" of a particular disorder and 5 - very intensive complaints.

\section{Mesopic contrast and glare sensitivity tests}

Mesopic contrast sensitivity was measured in the absence of glare and in the present of glare (i.e., glare sensitivity) using the apparatus Mesotest II (Oculus Germany). The subjects were allowed to have dark adaptation for at least $5 \mathrm{~min}$ before the examination [30]. The tests were conducted binocularly: 4 mesopic contrast sensitivity tests with different contrast levels (background luminance: $0.032 \mathrm{~cd} / \mathrm{m}^{2}$ ) and 4 glare sensitivity tests (glare source at the visual angle of $3^{\circ}$, background luminance: $0.1 \mathrm{~cd} / \mathrm{m}^{2}$ ). Four contrast levels $(1: 23,1: 5,1: 2.7,1: 2)$ of the Landolt's ring in 6 positions 
of the gap could be presented. The ring was displayed in a random position, and 5 rings were presented for each of the 8 tests. The presentation started from the higher contrast. The more correctly recognized rings, the better mesopic contrast sensitivity and the lower glare sensitivity.

The tests of mesopic contrast and glare sensitivity were carried out before the experimental session and just after the session. It was assumed that the significant difference between the correctly recognized rings before and after the experiment can be the evidence of bigger visual fatigue related to work performed under the modeled discomfort glare conditions.

\section{Statistical analysis}

The statistical analysis was performed using the SPSS 11.5 program. The nonparametric tests were used for the analysis of variables which represented the ordinal scale or for ratio variables which did not represent the normal distribution (according to the Kolmogorov-Smirnov test). Then, the median was the measure of the central tendency and 2 samples of related variables were estimated by the Wilcoxon Signed Ranks test, and of independent variables - by the Mann-Whitney U test. Statistical dependence between 2 variables was calculated by the Spearman's rank correlation coefficient.

The central tendency for the variables which represented the ratio scale (age, mistakes, time) was estimated by mean and standard deviation. In addition to this, the comparison between 2 samples of the ratio scale variables (independent) was done by the Mann-Whitney U test or the t-test, adequately to the results of the normality of the distribution tested by the Kolmogorov-Smirnov test. In this case, statistical dependence between 2 variables was calculated by the Pearson's rank correlation coefficient.

\section{RESULTS AND DISCUSSION}

\section{Discomfort glare evaluation}

For the purpose of analyzing the subjective evaluation of discomfort glare on the elaborated scale in relation to the UGR value, one of 13 UGR values (range: 16-30) for each mark on the scale was assigned (see Table 2).

The results of subjective discomfort glare assessment in both groups under the modeled glare conditions are presented in Figure 3 and Table 3. The tendency of bigger sensitivity to discomfort glare could be observed in the control group compared to the $50+$ group, which was manifested by a higher level of perceived discomfort on the glare rating scale (and higher UGR value).

Under both modeled glare conditions, bigger percentage of the control group subjects assessed glare after the experimental session as uncomfortable and intolerable than in the $50+$ group, in which the subjects more often assessed glare as acceptable (Figure 2).

Table 2. Semantic discomfort glare scale and unified glare rating (UGR) values ${ }^{1}$

\begin{tabular}{lc}
\hline \multicolumn{1}{c}{ Semantic discomfort glare scale } & UGR \\
\hline Intolerable & $29-30$ \\
Just intolerable & 28 \\
Between just uncomfortable and just intolerable (uncomfortable)* & $23-27$ \\
Just uncomfortable & 22 \\
Between just acceptable and just uncomfortable (acceptable)** & $17-21$ \\
Just acceptable & 16 \\
\hline
\end{tabular}

${ }^{1}$ Based on Berman et al. [10].

* Depending on the location of the mark on the visual analogue scale (VAS), more or less uncomfortable.

** Depending on the location of the mark on the VAS, more or less acceptable. 

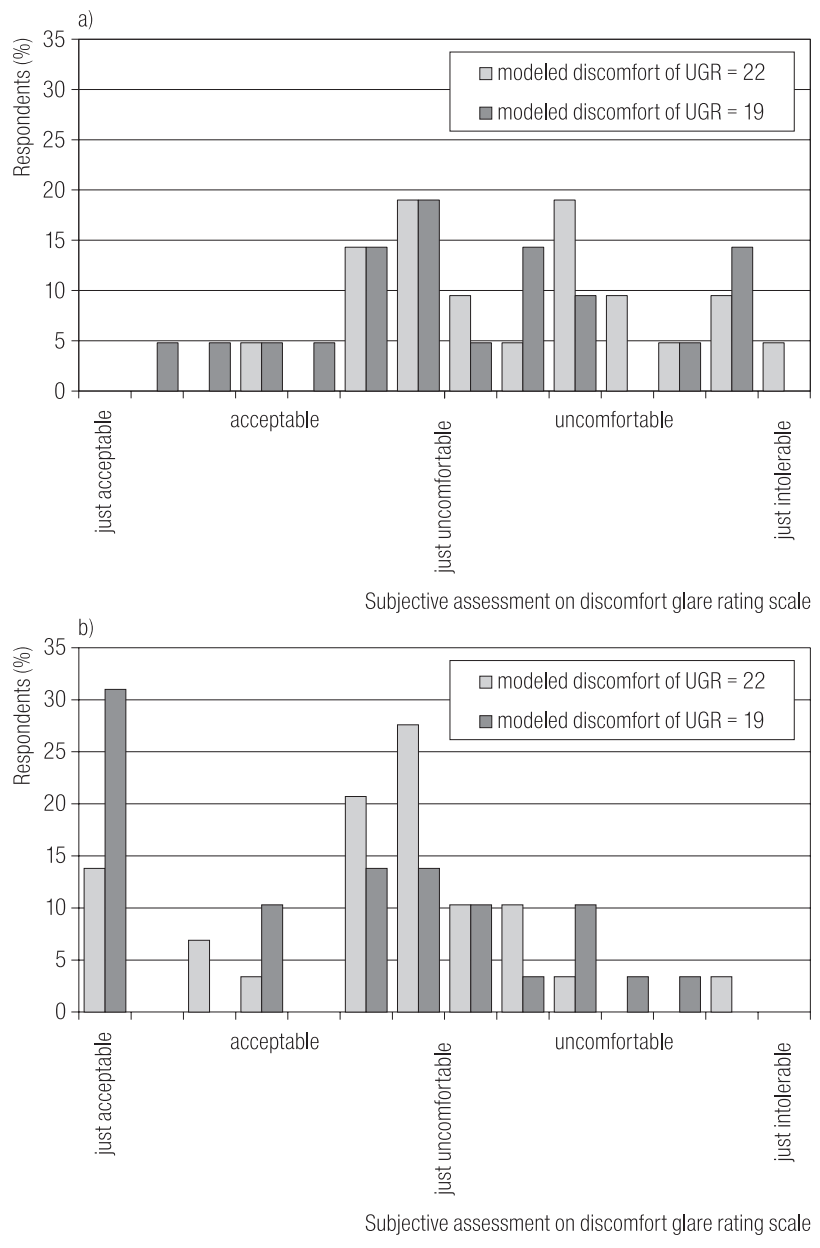

UGR - unified glare rating.

Fig. 2. Illustration of spread in discomfort glare assessment on the semantic scale under both modeled glare conditions:

a) in the control group, b) in the $50+$ group
It seems important to point out the median shift of the UGR assessment before and after the experiment (Table 3). In both groups and under both UGR conditions, there was observed significant lower perceived discomfort glare before the experiments. These results confirmed that the perception of discomfort glare depends on the exposure time and grows with time [7].

The control group glare assessment after the experiments under both glare conditions showed bigger glare sensation on the semantic scale. There can be observed a shift of 1 "semantic unit" for UGR = 19 (from acceptable to just uncomfortable) and of 3 "semantic units" for UGR $=22$ (from acceptable to uncomfortable). At the same time, in the 50+ group, there was no shift for UGR $=19$ (acceptable level on the semantic scale before and after the experiments) and a shift of 1 "semantic unit" was noted for UGR = 22 (from acceptable to just uncomfortable). However, significantly bigger discomfort glare perceived by younger subjects (the control group) was stated only for the experiment of lower modeled discomfort glare, i.e. $\mathrm{UGR}=19$ (Mann-Whitney test: $\mathrm{U}=196, \mathrm{p}=0.032$ ). In the experiment for UGR $=22$, the same tendency could be observed, but not statistically significant yet $(\mathrm{U}=221$, $\mathrm{p}=0.097)$.

It is worth pointing out that the subjective assessment of discomfort glare (on the semantic glare rating scale)

Table 3. Subjective evaluation of discomfort glare and corresponding unified glare rating (UGR) values according to the discomfort glare rating scale

\begin{tabular}{|c|c|c|c|c|}
\hline \multirow{2}{*}{ Group } & \multirow{2}{*}{ Modeled discomfort glare } & \multicolumn{2}{|c|}{ Median } & \multirow{2}{*}{$\begin{array}{l}\text { Wilcoxon Signed Ranks test } \\
\text { for UGR assessment of the } \\
\text { differences between before } \\
\text { and after the session } \\
\text { Z (2-tailed } p)\end{array}$} \\
\hline & & before session & after session & \\
\hline \multirow[t]{2}{*}{$50+$} & 19 (acceptable) & 17 (acceptable) & 21 (acceptable) & $-3.282(0.001)$ \\
\hline & 22 (just uncomfortable) & 17 (acceptable) & 22 (just uncomfortable) & $-3.679(<0.001)$ \\
\hline \multirow[t]{2}{*}{ Control } & 19 (acceptable) & 19 (acceptable) & 22 (just uncomfortable) & $-3.901(<0.001)$ \\
\hline & 22 (just uncomfortable) & 21 (acceptable) & 24 (uncomfortable) & $-2.656(0.008)$ \\
\hline
\end{tabular}

Z - Wilcoxon Signed Ranks test statistics. 
in the younger (control) group after both experiments corresponded to a higher UGR value compared to the UGR value calculated by DIALux. In the $50+$ group, higher subjective glare assessment compared to the calculated UGR value was found only for the lower modeled discomfort glare conditions.

\section{Subjective lighting quality assessment}

The results of the subjective assessment of the lighting showed that most participants $(80 \%)$ in both groups, under both glare conditions, assessed the illuminance level on the working plane (question 1) as sufficient or good.

Visual comfort related to lighting needed to perform a visual task (question 2) was assessed as uncomfortable by about half of the control group subjects $(47.6 \%)$ under both glare conditions, however, under UGR $=22$, the bigger percentage of subjects assessed it as definitely uncomfortable (19\%) compared to UGR $=19(4.8 \%)$. At the same time, in the $50+$ group, lighting was assessed as uncomfortable by $24.2 \%$ of the subjects in glare conditions of UGR $=19$, and by $34.4 \%$ in UGR $=22$ conditions.

The declaration of working under the modeled lighting conditions (question 3) showed that about half of the subjects in both groups declared "sometimes", and the remaining participants in the control group declared "rarely/ occasionally" or "never", while in the 50+ group - "often" or "always".

The assessment of glare sources appearance (question 4) showed that about half of the subjects in the control group under both glare conditions assessed them as "medium bright and of big size" and $28.5 \%$ as "very bright and of big size" (which was related to number 1 on the ordinal scale) and "very bright and of medium size" (number 2 on the ordinal scale). Simultaneously, $17.2 \%$ of the subjects in the $50+$ group under both glare conditions assessed glare sources as "medium bright and of big size" and $10.2 \%$ as "very bright and of big size" and "very bright and of medium size".

The question concerning permanent work under the modeled lighting (question 5) showed that most subjects from the control group in both glare conditions $(71.4 \%$ for UGR $=19,85.6 \%$ for UGR $=22$ ) would not have liked to work under these conditions all the time.

The medians of answers to particular questions in both groups are presented in Table 4. There were noted statistical differences of the lighting quality assessment between the groups for both glare conditions in the answers to questions: 2, 3, 4 and 5 (Mann-Whitney U test). The significant difference of answers to question 4 in the $50+$ group between both glare conditions was found. The older $(50+)$ group perceived the glare source to be of a smaller size than the younger (control) group.

The assessment of lighting quality did not depend on the modeled discomfort glare, but on the subjects' age.

\section{Subjective visual fatigue assessment}

The results of the subjective assessment of visual fatigue showed that younger participants (control group) more frequently suffered from asthenopic symptoms. Most subjects reported: tiredness $(100 \%$ subjects in the control group, $62-82 \%$ - in the $50+$ group), oversensitivity to light (81-91\% subjects in the control group, $70-83 \%$ - in the $50+$ group), redness (33-52\% subjects in the control group, $81-83 \%$ - in the $50+$ group), blurring (71-81\% subjects in the control group, 41-69\% - in the 50+ group), eyelids heaviness (86-91\% subjects in the control group, 38-70\% - in the 50+ group). The medians of intensity of particular asthenopic symptoms in both groups under the modeled discomfort glare conditions are presented in Table 5.

There were no statistical differences of visual fatigue symptoms complaints between experiments for both groups. Nevertheless, there were found significant differences (Mann-Whitney U test) between both groups 
Table 4. Subjective evaluation of the lighting quality in both groups under the modeled discomfort glare conditions (ordinal scale: $1-5$ )

\begin{tabular}{|c|c|c|c|c|c|}
\hline \multirow{3}{*}{ Question } & \multirow{3}{*}{$\begin{array}{l}\text { Feature of lighting } \\
\text { assessment }\end{array}$} & \multicolumn{4}{|c|}{ Median / related assessment } \\
\hline & & \multicolumn{2}{|c|}{$50+$ group } & \multicolumn{2}{|c|}{ control group } \\
\hline & & UGR $=19$ & UGR $=22$ & $\mathrm{UGR}=19$ & UGR $=22$ \\
\hline 1 & $\begin{array}{l}\text { illuminance of the } \\
\text { working plane }\end{array}$ & 4 (good) & 3 (sufficient) & 3 (sufficient) & 3 (sufficient) \\
\hline 2 & $\begin{array}{l}\text { visual comfort related } \\
\text { to lighting }\end{array}$ & 3 (sufficient) & 3 (sufficient) & 2 (uncomfortable) & 2 (uncomfortable) \\
\hline 3 & $\begin{array}{l}\text { declaration of } \\
\text { working under the } \\
\text { modeled lighting } \\
\text { conditions }\end{array}$ & 3 (sometimes) & 3 (sometimes) & 3 (sometimes) & 2 (rarely/occasionally) \\
\hline 4 & $\begin{array}{l}\text { assessment of glare } \\
\text { sources appearance }\end{array}$ & $\begin{array}{l}4 \text { (medium bright } \\
\text { and of small size) }\end{array}$ & $\begin{array}{l}4 \text { (medium bright } \\
\text { and of small size) }\end{array}$ & $\begin{array}{l}3 \text { (medium bright } \\
\text { and of big size) }\end{array}$ & $\begin{array}{l}3 \text { (medium bright } \\
\text { and of big size) }\end{array}$ \\
\hline 5 & $\begin{array}{l}\text { possibility of } \\
\text { permanent work } \\
\text { under the modeled } \\
\text { lighting }\end{array}$ & 3 (no matter) & 2 (rather no) & 2 (rather no) & 2 (rather no) \\
\hline
\end{tabular}

UGR - unified glare rating.

Table 5. Subjective evaluation of visual fatigue in both groups under the modeled discomfort glare conditions

\begin{tabular}{lcccc}
\hline \multirow{2}{*}{ Symptom } & \multicolumn{4}{c}{ Median / intensity of complaint } \\
\cline { 2 - 5 } & \multicolumn{3}{c}{$50+$ group } & \multicolumn{2}{c}{ control group } \\
\cline { 2 - 5 } & UGR $=19$ & UGR $=22$ & UGR $=19$ & UGR $=22$ \\
\hline Itching & 1 & 1 & 1 & 1 \\
Tearing & 1 & 2 & 1 & 2 \\
Shooting pain & 1 & 1 & 2 & 1 \\
Gritty eyes & 1 & 2 & 2 & 1 \\
Oversensitivity to light & 2 & 2 & 3 & 3 \\
Heaviness of eyelids & 1 & 1 & 3 & 3 \\
Blurring & 2 & 2 & 3 & 3 \\
Visual tiredness & 3 & 3 & 3 & 4 \\
Headache & 1 & 1 & 1 & 1 \\
Redness & 2 & 2 & 1 & 2 \\
\hline
\end{tabular}

UGR - unified glare rating. Ordinal scale: 1 - no complaint; 2 - little; 3 - medium; 4 - big.

under the studied discomfort conditions for the following symptoms: heaviness of eyelids ( $\mathrm{UGR}=19$ : $\mathrm{U}=145.5$, $\mathrm{p}=0.002$; UGR $=22: \mathrm{U}=129, \mathrm{p}<0.001)$, visual tiredness $(\mathrm{UGR}=22$ : $\mathrm{U}=204.5, \mathrm{p}=0.041)$, and redness
$(\mathrm{UGR}=19: \mathrm{U}=196.5, \mathrm{p}=0.016 ; \mathrm{UGR}=22: \mathrm{U}=203$, $\mathrm{p}=0.027$ ).

Visual fatigue manifested by different asthenopic symptoms did not depend on the modeled discomfort glare, but 
on the subjects' age. The younger (control) group reported bigger visual fatigue than the older $(50+)$ group and more frequently.

\section{Mesopic contrast and glare sensitivity}

The numbers of correctly recognized Landolt's rings both in mesopic contrast sensitivity tests and glare sensitivity tests before and after the experiments for both groups and under both modeled glare conditions are presented in Table 6.

Significant differences for mesopic contrast sensitivity before and after the experimental session were found for the younger (control) group in the experiment of higher discomfort glare $(\mathrm{UGR}=22)$ and for the older $(50+)$ group in the experiment of lower discomfort glare $(\mathrm{UGR}=19)$. Significant differences of glare sensitivity before and after the experimental session were stated only for the older group in both glare conditions. However, there were no statistically significant differences between mesopic contrast sensitivity and glare sensitivity between the experiments under different discomfort glare conditions among both groups.

The results of the Mann-Whitney U test pointed out the significant differences of both mesopic parameters (measured after the experimental session) between the groups: contrast sensitivity ( $U G R=22: \mathrm{U}=180.5, \mathrm{p}=0.014)$, glare sensitivity $(\mathrm{UGR}=19: \mathrm{U}=134.5, \mathrm{p}=0.001$; UGR $=21: 155.5$, $p=0.003)$. The mesopic glare sensitivity significantly increased after the experiments (lower number of correctly recognized rings) only in the $50+$ group under both glare conditions. The smaller the number of correctly recognized rings, the bigger discomfort glare.

\section{Visual performance}

Visual performance was evaluated on the basis of the number of mistakes and the time needed to perform the visual task. The results of visual performance obtained for both groups and under both modeled glare conditions are presented in Table 7.

No significant differences in the time needed to perform the visual task and the number of mistakes between the experiments of different glare conditions were found in both groups. The results of the Mann-Whitney U test pointed out the significant differences of visual performance parameters between the groups: the time needed to perform the visual task: (UGR $=19$ : $\mathrm{U}=101, \mathrm{p}<0.001$; $\mathrm{UGR}=22: \mathrm{U}=160.5, \mathrm{p}=0.005)$, the number of mistakes $(\mathrm{UGR}=19: \mathrm{U}=42, \mathrm{p}<0.001 ; \mathrm{UGR}=21: \mathrm{U}=6$, $\mathrm{p}<0.001)$. The participants from the $50+$ group performed the visual task significantly longer and made

Table 6. Correctly recognized Landolt's rings in mesopic contrast sensitivity tests and glare sensitivity tests before and after the experiments in both groups under the modeled discomfort glare conditions

\begin{tabular}{|c|c|c|c|c|c|c|c|}
\hline \multirow{3}{*}{ Group } & \multirow{3}{*}{$\begin{array}{l}\text { Modeled } \\
\text { discomfort } \\
\text { glare }\end{array}$} & \multicolumn{6}{|c|}{ Correctly recognized Landolt's rings } \\
\hline & & \multicolumn{3}{|c|}{ mesopic contrast sensitivity tests } & \multicolumn{3}{|c|}{ glare sensitivity tests } \\
\hline & & $\begin{array}{c}\text { before } \\
(\mathrm{M} \pm \mathrm{SD})\end{array}$ & $\begin{array}{c}\text { after } \\
(\mathrm{M} \pm \mathrm{SD})\end{array}$ & statistics (2-tailed p) & $\begin{array}{c}\text { before } \\
(\mathrm{M} \pm \mathrm{SD})\end{array}$ & $\begin{array}{c}\text { after } \\
(\mathrm{M} \pm \mathrm{SD})\end{array}$ & $\begin{array}{c}\text { statistics } \\
\text { (2-tailed p) }\end{array}$ \\
\hline \multirow[t]{2}{*}{$50+$} & $\mathrm{UGR}=19$ & $14.2 \pm 6.5$ & $13.0 \pm 7.1$ & $\mathrm{t}=3.123(\mathrm{p}=0.004)$ & $10.0 \pm 3.4$ & $8.4 \pm 7.5$ & $\mathrm{t}=2.539(\mathrm{p}=0.017)$ \\
\hline & $\mathrm{UGR}=22$ & $14.1 \pm 6.3$ & $12.3 \pm 6.5$ & $\mathrm{t}=1.509(\mathrm{p}=0.142)$ & $9.4 \pm 7.3$ & $7.8 \pm 6.7$ & $\mathrm{t}=3.053(\mathrm{p}=0.005)$ \\
\hline \multirow[t]{2}{*}{ Control } & $\mathrm{UGR}=19$ & $16.5 \pm 5.6$ & $16.3 \pm 5.9$ & $\mathrm{Z}=-0.951(\mathrm{p}=0.342)$ & $15.2 \pm 6.8$ & $15.2 \pm 7.0$ & $\mathrm{Z}=-0.032(\mathrm{p}=0.975)$ \\
\hline & $\mathrm{UGR}=22$ & $18.2 \pm 3.4$ & $16.2 \pm 5.2$ & $Z=-2.747(p=0.006)$ & $15.3 \pm 6.3$ & $13.8 \pm 7.5$ & $\mathrm{t}=1.418(\mathrm{p}=0.172)$ \\
\hline
\end{tabular}

Analysis of differences between before and after the measurements was made using the Wilcoxon Signed Ranks test $(\mathrm{Z})$ or $\mathrm{t}$-test ( $\mathrm{t})$, depending on the normality of distribution.

Abbreviations as in Tables 1 and 4. 
Table 7. Visual performance in both groups of participants under the modeled discomfort glare conditions

\begin{tabular}{|c|c|c|c|c|}
\hline \multirow{3}{*}{ Feature } & \multicolumn{4}{|c|}{ Parameters of visual performance } \\
\hline & \multicolumn{2}{|c|}{$50+$ group } & \multicolumn{2}{|c|}{ control group } \\
\hline & $\mathrm{UGR}=19$ & $\mathrm{UGR}=22$ & $\mathrm{UGR}=19$ & $\mathrm{UGR}=22$ \\
\hline \multicolumn{5}{|c|}{$\begin{array}{l}\text { Time needed to perform } \\
\text { the visual task (min) }\end{array}$} \\
\hline $\mathrm{M} \pm \mathrm{SD}$ & $67.17 \pm 8.7$ & $65.1 \pm 8.77$ & $57.4 \pm 6.77$ & $57.95 \pm 6.7$ \\
\hline $\min .-\max$ & $55-87$ & $50-84$ & 44-71 & 45-69 \\
\hline \multicolumn{5}{|l|}{ Mistakes (n) } \\
\hline $\mathrm{M} \pm \mathrm{SD}$ & $143.8 \pm 71.15$ & $128 \pm 28.34$ & $56 \pm 24.5$ & $51.71 \pm 18.66$ \\
\hline $\min .-\max$ & $60-321$ & 68-198 & $25-116$ & $19-99$ \\
\hline
\end{tabular}

Abbreviations as in Table 1 and 4.

Table 8. Correlations between age and visual fatigue assessment, visual performance, lighting quality assessment and mesopic sensitivities $(\mathrm{N}=100)$

\begin{tabular}{lc}
\hline \multicolumn{1}{c}{ Variable } & $\begin{array}{c}\text { Age vs. variable } \\
\mathrm{r}(2 \text {-tailed p })\end{array}$ \\
\hline Heaviness of eyelids & $-0.440(<0.001)^{*}$ \\
Redness & $0.432(<0.001)^{*}$ \\
Time needed to perform the visual task & $0.499(<0.001)^{* *}$ \\
Number of mistakes & $0.743(<0.001)^{* *}$ \\
Comfortable lighting & $0.257(0.010)^{*}$ \\
Working under the modeled lighting conditions & $0.368(<0.001)^{*}$ \\
Annoyance related to bright light sources & $0.295(0.003)^{*}$ \\
Lighting acceptance for permanent work & $0.248(0.013)^{*}$ \\
Mesopic contrast sensitivity & \\
$\quad$ before & $-0.277(0.005)^{* *}$ \\
after & $-0.288(0.004)^{* *}$ \\
Glare sensitivity & \\
before & $-0.396(<0.001)^{* *}$ \\
after & $-0.448(<0.001)^{* *}$ \\
\hline
\end{tabular}

$\mathrm{r}$ - correlation coefficient: * Spearman's rank correlation; ** Pearson's correlation.

significantly more mistakes than the younger subjects viness of eyelids, redness), answers to questions 2-5 relafrom the control group.

\section{Correlations}

There were found correlations between the age of participants and: visual performance, 2 asthenopic symptoms (heated to lighting quality assessment, mesopic contrast sensitivity and glare sensitivity. The results are presented in Table 8.

The biggest correlations were found between age and visual performance parameters, which showed that visual 
performance decreases with age. The correlation between age and asthenopic symptoms and lighting quality assessment revealed that older participants assessed lighting as more comfortable, complained of heaviness of eyelids less often, but had bigger redness.

It is noteworthy that the obtained results of lower mesopic contrast sensitivity and bigger glare sensitivity measured before the experiment among the older $(50+)$ group (see Table 6) and theirs correlations with age (see Table 8) are consistent with other studies which found mesopic contrast and glare sensitivity deteriorated in an agedependent way [30,31]. The mesopic contrast sensitivity decreased gradually from 51 to 60 years of age onwards, whereas glare sensitivity started to decline at an earlier age (41-50 years) [30].

Small or medium correlations were found between visual performance and mesopic contrast sensitivity and glare sensitivity (Table 9). The longer the time needed to perform the task and the bigger the number of mistakes, the lower the number of correctly recognized rings for both mesopic sensitivities.

No correlations were found for discomfort glare assessment after the experiment and visual performance parameters or the age of the participants. However, there were correlations between discomfort glare assessment after the experiment and some asthenopic symptoms: oversensitivity to light: $\mathrm{r}=0.656, \mathrm{p}<0.001$, visual tiredness: $r=0.545, p<0.001$, blurring: $r=0.474, p<0.001$, shooting pain: $r=0.268, p=0.007$, heaviness of eyelids: $r=0.268, p=0.007$, gritty eyes: $r=0.257, p=0.010$, and headache: $r=0.212, p=0.034$.

There were also noted correlations between discomfort glare assessment after the experiment and answers to questions concerned the lighting quality assessment: question 1: $r=-0.417, p<0.001$, question $2: r=-0.576$, $\mathrm{p}<0.001$, question $3: \mathrm{r}=-0.570, \mathrm{p}<0.001$, question 4 : $r=-0.458, p<0.001$, question $5: r=-0.567, p<0.001$. These results show that higher perceived discomfort glare sensation increased with bigger visual complaints (especially oversensitivity to light) and worse lighting assessment.

\section{CONCLUSIONS}

The obtained results for the modeled glare of UGR $=19$ and UGR $=22$ showed that discomfort glare sensation changes with age.

In the subjective assessment, the level of discomfort glare one a computer work station was higher in the control group compared with the 50+ group, which suggests that from the psychological point of view, younger people are more sensitive to discomfort glare than older people. However, the difference of discomfort glare sensation between both groups seems to be of less significance with the increasing modeled glare.

Although subjective assessments of 50+ participants (regarding discomfort glare on the semantic scale, visual fatigue and lighting quality) demonstrated less negative

Table 9. Correlations between visual performance parameters and mesopic contrast sensitivity and glare sensitivity before and after the experiment $(\mathrm{N}=100)$

\begin{tabular}{lcccc}
\hline \multirow{2}{*}{ Feature } & \multicolumn{4}{c}{$\mathrm{r}(2$-tailed $\mathrm{p})$} \\
\cline { 2 - 4 } & \multicolumn{2}{c}{ mesopic contrast sensitivity } & gefore & after \\
\cline { 2 - 4 } & before & after & bensitivity \\
\hline $\begin{array}{l}\text { Time needed to perform } \\
\text { the visual task }\end{array}$ & $-0.223(0.026)$ & not significant & $-0.254(0.011)$ & $-0.229(0.022)$ \\
Number of mistakes & $-0.248(0.013)$ & $-0.332(0.001)$ & $-0.324(0.001)$ & $-0.395(<0.001)$ \\
\hline
\end{tabular}

r - Pearson's correlation coefficient. 
psychological sensation related to glare impact, compared with control group, the exposure for about 65 minutes to bigger modeled discomfort glare caused the decrease of theirs visual performance and increase of mesopic glare sensitivity. It means that exposure of the elderly to bigger discomfort glare could adversely affect the objective measures of fatigue like mesopic glare sensitivity (i.e., disability glare sensitivity) and visual performance.

On the other hand, subjective evaluations of participants in the control group indicated higher, than in the 50+ group, sensitivity to discomfort glare on the semantic scale, bigger visual fatigue and worse lighting quality assessment after about 58-minute exposure to both modeled discomfort glare conditions. Nevertheless, the adverse effect of the modeled discomfort glare on theirs mesopic glare sensitivity and visual performance was not noted. From the psychological point of view, the younger population seems to be more sensitive and demanding than the older one in relation to discomfort glare limiting, in spite of the lack of significant changes in objective measures of fatigue.

The obtained results confirmed the importance of discomfort glare limitation, regardless of the age of the users. Although the $50+$ group complained less on the lighting quality and discomfort glare than the control group, exposure to glare had a greater effect on their fatigue manifested by bigger mesopic glare sensitivity and lower work efficiency. It means that discomfort glare for both user groups should be limited more at the designing stage. The UGR values calculated by DIALux should be lower by 1 to 3 UGR units than the glare limit value required by the lighting standard [17]. It is advisable both for the well-being or health of employees and for the employers' interest related to better work performance and job satisfaction.

On the other hand, the differences between subjective discomfort glare assessment and UGR values calculated by DIALux raise questions. Could the reason for these differences be the accuracy of UGR calculations by DIALux? It is an important aspect both for lighting designing and assessment. Will discomfort glare be really limited if the UGR value calculated by DIALux for particular lighting installation fulfills the standard's requirements for UGR limit value? This suggests that another objective method of discomfort glare assessment or measurement is needed. One of the currently developed approaches to objective evaluation of UGR is measurement of luminance distribution in the visual field using the matrix system where a digital camera is used [32].

Further studies are needed to establish the border of discomfort glare and the related UGR value at which there are no differences of glare sensation between the young and the elderly. It is assumed that at higher levels of discomfort glare of UGR $\geq 25$, the existing glare could be annoying to such an extent that regardless of age it would be assessed very similar.

\section{REFERENCES}

1. Preamble to the Constitution of the World Health Organization as adopted by the International Health Conference, New York, 19-22 June, 1946; signed on 22 July 1946 by the representatives of 61 States (Official Records of the World Health Organization, no. 2, p. 100) and entered into force on 7 April 1948. Geneva, Switzerland [cited 2013 Oct 1]. Available from: http://www.who.int/about/definition/en/print. html.

2. Veitch JA. Lighting for well-being: A revolution in lighting? Proceedings of the 2nd CIE Expert Symposium on Lighting and Health (CIE x031:2006); 2006 Sep 7-8; Ontario, Canada. Vienna: Commission Internationale de L'Ecalirage; 2006. p. 56-61.

3. Commission Internationale de L'Ecalirage. Ocular lighting effects on human physiology and behavior. Vienna: CIE; 2004. CIE technical report no. 158.

4. Aschoff J. Handbook of behavioral neurobiology, biological rhythms. New York: Plenum Press; 1981. 
5. Rea MS. Lighting handbook reference \& application. 9th ed. New York: Illuminating Engineering Society of North America; 2000.

6. Veitch J, Newsham R. Determinants of lighting quality II: Research and recommendations. Ottawa: National Research Council of Canada; 1996. p. 1-17.

7. Commission Internationale de L'Ecalirage. Discomfort glare in the interior working environment. Vienna: CIE; 1983. CIE Publication no. 55 (TC 3.4).

8. Wolska A. Glare as a specific factor in the working environment. Przegl Elektrotechn. 2013;89(1a/2013):142-4.

9. Hopkinson RG. Glare from daylighting in buildings. Appl Ergon. 1972;3:206-15, http://dx.doi.org/10.1016/0003-6870 (72)90102-0.

10. Berman SM, Bullimore MA, Jacobs RJ, Bailey LL, Gandhi N. An objective-measure of discomfort glare. J Illuminat Eng. 1994;23:40-9, http://dx.doi.org/10.1080/00994480.1994. 10748079 .

11. Bennet CA. Discomfort glare, the effect of instructions. Special report \#103. Manhattan: Kansas State University Engineering Experiment Station; 1972.

12. Clarke KC, Boyce PR, Beckstead JW. Factors influencing the stability of glare assessments. Proceedings of the Conference of the Illuminating Engineering Society of North America; 1991 Aug 11-15; Montreal, Canada. New York: Illuminating Engineering Society of North America; 1991. p. 36-66.

13. Eble-Hankins M. Subjective impression of discomfort glare from sources of non-uniform luminance [dissertation and student research paper] [Internet]. University of Nebraska of Lincoln Architectural Engineering; 2008 [cited 2013 Oct 1]. Available from: http://digitalcommons.unl.edu/cgi/ viewcontent . cgi? article $=1000 \&$ context $=$ archengdiss.

14. Geedrinck L. Glare perception in terms of acceptance and comfort [graduation report]. Eindhoven: University of Technology; 2012.

15. Hopkinson RG. Evaluation of glare. Illum Eng. 1957;52(6):305-16.
16. Commission Internationale de L'Ecalirage. Lighting of work places part 1: Indoor. Vienna: CIE; 2001. CIE Publication S 008/E;2001.

17. European Committee for Standardization. Light and lighting. Part 1. Lighting of work places. Indoor work places. Brussels: European Committee for Standardization; 2011. European Standard EN 12464-1.

18. Wolska A, Sawicki S. Comparison of discomfort glare evaluation using different techniques. Proceedings of 12th European Lighting Conference LUX EUROPA 2013; 2013 Sep 17-19; Kraków, Poland. Warszawa: Polish Committee on Illumination (CIE Poland); 2013. p. 553-8.

19. Commission Internationale de L'Ecalirage. Discomfort glare in interior lighting. Vienna: CIE; 1995. CIE Publication no. 117 (TC 3-13) 1995.

20. Gross Figueiro M. Lighting the way: A key to independence. Lighting Research Center at Rensselaer Polytechnic Institute: New York; 2001 [cited 2013 Oct 1]. Available from: http://www.Irc.rpi.edu/programs/lightHealth/AARP/pdf/ AARPbook1.pdf.

21. Nersveen J. Sight weakness and universal design. Proceedings of CIE 26th Session; 2007 Jul 4-11; Beijing, China. Vienna: Commission Internationale de L'Ecalirage (CIE); 2007. p. D3-77.

22. Van de Kraatz J, van Norren D. Optical density of the aging human ocular media in the visible and the UV. J Opt Soc Am A. 2007;24(7):1842-57, http://dx.doi.org/10.1364/JOSAA.24.001842.

23. Pinto MR, Medici S, Zlotnicki A, Bianchi A, Sant C, Napoli C. Reduced visual acuity in elderly people: The role of ergonomics and geronotechnology. Age Ageing. 1997;(26):339-44, http://dx.doi.org/10.1093/ageing/26.5.339.

24. Schierz C. Lighting for the elderly: Physiological basics and their consequences. Proceedings of the Conference LUX EUROPA 2009; 2009 Sep 9-11; Istanbul. Istanbul: Turkish National Committee on Illumination (ATMK); 2009. p. $147-54$. 
25. Geerdinck L, Zande B, Talen A, Erp T, Schlangen L. Comfortable lighting for seniors: The effect of illuminance and color temperature on the visual acuity in later life. Proceedings of the Conference LUX EUROPA 2009; 2009 Sep 9-11; Istanbul. Istanbul: Turkish National Committee on Illumination (ATMK); 2009. p. 357-62.

26. Moosmann C, Wienold J, Wagner A, Wittwer V. Age effects on glare perception under daylight conditions. Proceedings of the Conference LUX EUROPA 2009; 2009 Sep 9-11; Istanbul. Istanbul: Turkish National Committee on Illumination (ATMK); 2009. p. 439-42.

27. DIALux [Internet]. DIAL light. Building. Software [cited 2013 May 15]. Available from: http://www.dial.de/DIAL/ en/dialux.html.

28. Wolska A. Visual strain and lighting preferences of VDT users under different lighting systems. Int J Occup Saf Ergon. 2003;9(4):431-40.
29. Wolska A. Intelligent lighting systems and users' visual fatigue. Przegl Elektrotechn. 2007;83(5):97-9.

30. Puell MC, Palomo C, Sanchez-Ramos C, Villena C. Mesopic contrast sensitivity in the presence or absence of glare in a large driver population. Graefes Arch Clin Exp Ophthalmol. 2004 [cited 2013 Oct 1];242(9):755-61. Available from: http://www.oculus.de/pl/sites/detail_ger.php?page =525.

31. Scharwey K, Krzizok T, Herfurth M. Night driving capacity of ophthalmologically healthy persons of various ages Ophthalmologe. 1998;95:555-8, http://dx.doi.org/10.1007/ s003470050315.

32. Sawicki S, Wolska A. Algorithm of HDR image preparation for discomfort glare assessment. Przegl Elektrotechn. 2013;89(2a):87-90.

This work is available in Open Access model and licensed under a Creative Commons Attribution-NonCommercial 3.0 Poland License - http://creativecommons.org/ licenses/by-nc/3.0/pl/deed.en. 